\title{
Comparison of Two Static Stretching Techniques for the Triceps Surae in Healthy Individuals: Wall and Inclined Board Stretchings
}

\author{
Tae Hee Kim, MD, PhD ${ }^{1}$, Oh Kyung Lim, MD, PhD², Ki Deok Park, MD, PhD², Ju Kang Lee, MD, PhD \\ ${ }^{1}$ Department of Rehabilitation Medicine, Konkuk University Chungju Hospital, Chungju; \\ ${ }^{2}$ Department of Rehabilitation Medicine, Gil Medical Center, Gachon University College of Medicine, Incheon, Korea
}

Objective To compare the effectiveness of static stretching techniques for correcting the tightness of the triceps surae.

Methods In this observational, cross-sectional study, participants (30 healthy volunteers) completed 10 repetitions of each stretching exercise, holding each stretch for 10 seconds, with a 1-minute rest period between repetitionsand a 1-hour rest period between the two stretching techniques, namely, wall and inclined board stretchings. The length of the triceps surae and range of ankle dorsiflexion were measured on lateral view radiographs. The muscle activity during the stretch was measured using quantified surface electromyography of the lateral gastrocnemius. The subjective stretching sensation was evaluated using the visual analog scale.

Results Both stretching techniques showed statistical differences in all the parameters. Stretching on an inclined board yielded a greater increase in the triceps surae length than did wall stretching (mean difference, 0.72; $\mathrm{p}=0.02$ ). The range of ankle dorsiflexion was higher with inclined board stretching than with wall stretching (mean difference, 2.57; $\mathrm{p}=0.03$ ). The mean muscle activity was significantly lower withinclined board stretching than with wall stretching (mean difference, 53.72; $\mathrm{p}<0.01$ ). The visual analog scale score was higher with inclined board stretching than with wall stretching (mean difference, $2.07 ; \mathrm{p}<0.01$ ).

Conclusion In this study, inclined board stretching was more effective than wall stretching for correcting tightness of the triceps surae. Therefore, inclined board stretching should be encouraged for the triceps surae.

Keywords Achilles tendon, Inclined board, Triceps surae, Wall stretch

\footnotetext{
Received July 12, 2019; Revised August 29, 2019; Accepted September 10, 2019

Corresponding author: Ju Kang Lee

Department of Rehabilitation Medicine, Gil Medical Center, Gachon University College of Medicine, 21 Namdong-daero 774 Beon-gil, Namdong-gu, Incheon 21565, Korea. Tel: +82-32-458-2734, Fax: +82-32-460-3722, E-mail: pmrdoc@gilhospital.com

ORCID: Tae Hee Kim (https://orcid.org/0000-0002-9141-6380); Oh Kyung Lim (https://orcid.org/0000-0002-4286-8073); Ki Deok Park (https://orcid. org/0000-0003-1684-4737); Ju Kang Lee (https://orcid.org/0000-0002-8335-9785).
}

(c) This is an open-access article distributed under the terms of the Creative Commons Attribution Non-Commercial License (http://creativecommons.org/ licenses/by-nc/4.0) which permits unrestricted noncommercial use, distribution, and reproduction in any medium, provided the original work is properly cited. Copyright $\odot 2020$ by Korean Academy of Rehabilitation Medicine 


\section{INTRODUCTION}

Tightness of the triceps surae can be asymptomatic, but its prevalence accounts for up to $96.5 \%$ in patients with pathological symptoms that affect the feet and ankles [1]. It frequently causes limited ankle dorsiflexion and is associated with common foot and ankle pathologies such as Achilles tendinitis and plantar fasciitis $[2,3]$. As such, interest has emerged in the development ofthe most effective stretching program for the triceps surae, both for injury prevention and treatment, in clinical practice $[2,4,5]$.

Regular stretching of the gastrocnemius and soleus muscles (and of their tendons) is generally recommended as a low-risk intervention to increase flexibility in patients with shortened connective tissues or to maintain ankle dorsiflexion range of motion (ROM) in healthy individuals [5]. Several stretching methods are adopted to increase flexibility, including passive, static, isometric, and ballistic stretching techniques, and proprioceptive neuromuscular facilitation [6]. However, the most appropriate stretching technique for the triceps surae remains controversial $[6,7]$.

Two basic static stretching techniques are commonly used in clinical practice, namely wall and inclined surface stretchings. Wall stretching is performed by placing the foot at a distance from the wall, with the subject leaning forward, keeping the knee in extension, which leads to stretching of the triceps surae [8]. The other technique is stretching on an inclined board. Standing on the inclined surface induces dorsiflexion of the ankle joint, which leads to the stretching of the triceps surae [9]. To date, these two basic stretching techniques have not been compared in terms of their effectiveness in improving the tightness of the triceps surae. Therefore, to elucidate this point, this study aimed to compare the effectiveness of inclined board and wall stretchings in healthy individuals.

\section{MATERIALS AND METHODS}

\section{Participants}

This study was approved by the Institutional Review Board of Gachon University Gil Hospital (No. GIRBD0032-2012), and all the participants provided informed consent. The participants' eligibility to partici- pate in the study was assessed using a questionnaire after the participants signed up for the research. The study group included 30 healthy volunteers with no history of injury, or structural and physiological abnormalities of the lower limbs. Individuals with histories of systemic inflammatory disease (e.g., rheumatic disease, gout, and ankylosing spondylosis), prior lower limb surgery, congenital and/or acquired lower limb deformity, and a restriction in ankle ROM due to trauma (e.g., fracture) and local inflammation (e.g., Achilles tendonitis or plantar fasciitis) were excluded [10].

\section{Protocol}

Before randomization, the participants were screened to ensure accurate inclusion or exclusion, and their age, sex, height, and weight were recorded. The participants received instruction on how to perform the two stretching techniques. Each stretch was held for 10 seconds, with 10 repetitions of each stretch completed with a 1-minute rest period between repetitions. Outcome variables were measured during the repetitions, with a 1-hour rest period provided between the two exercises, on the basis of a previously published methodology [11] (Fig. 1).

Both static stretching techniques are used clinically

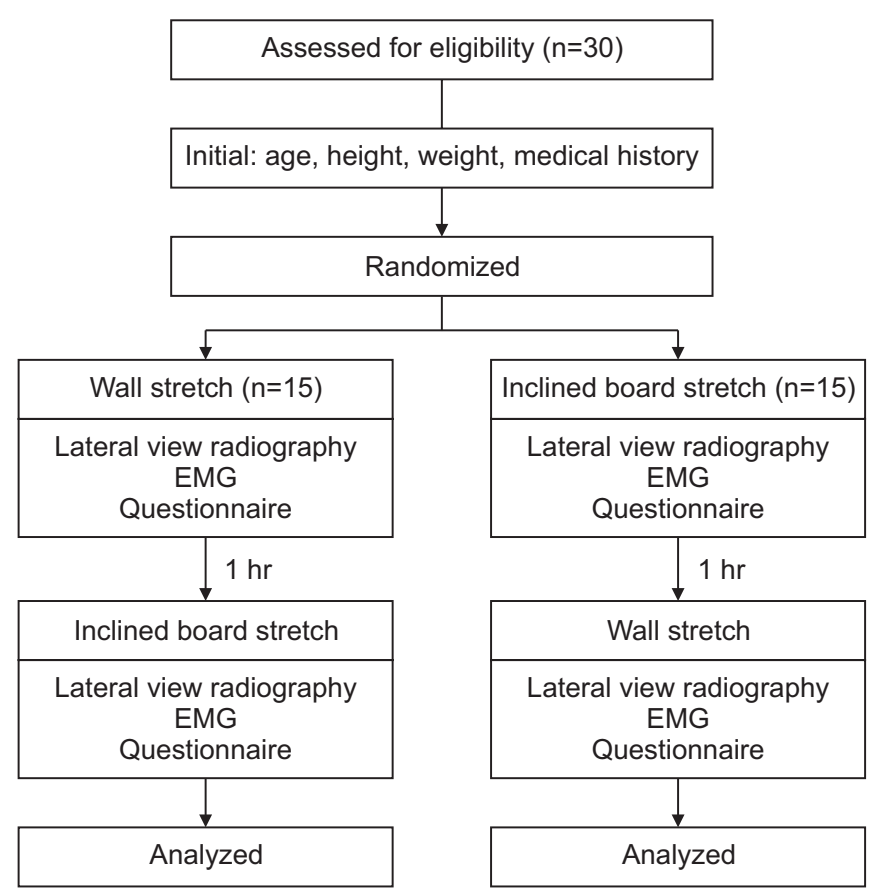

Fig. 1. Flow of participants through the study. EMG, electromyography. 
todecrease the tightness of the triceps surae $[8,9]$. For the wall stretch (Fig. 2), the participants were asked to stand facing a wall, with the lower limb to be stretched extended behind and the contralateral leg placed in front. Keeping the trunk in an upright position, the participants were asked to flex the knee of their front leg until they felt a stretch in the triceps surae of the back leg, keeping the heel on the ground during the stretch. By following a previously published method [10], the participants were asked to use a "toe in" position, with the toes of the back foot pointing toward the heel of the front foot. For stretching on an inclined board, a standard board was used, with a width of $27.5 \mathrm{~cm}$, length of $11 \mathrm{~cm}$, and height of $7.5 \mathrm{~cm}$, forming an inclination angle of $30^{\circ}$. The participants were asked to stand with both feet on the inclined board, with the heels placed at the lower edge of the board [9]. They were instructed to lean forward with their hands on the wall and then to bend their elbows until they felt a stretch in the triceps surae bilaterally (Fig. 3).

To minimize the effects of anticipation of the results on the measured outcomes, the participants were only instructed on the methods of stretching and outcome measurement, with no information regarding the anticipated effect of the stretching. In addition, in the registered order of the test subjects, the order of the two exercises was progressed alternately. An independent researcher not involved in the study enrolled the participants. The participants were asked to avoid intense exercise before the testing session that could cause muscle fatigue and influ-

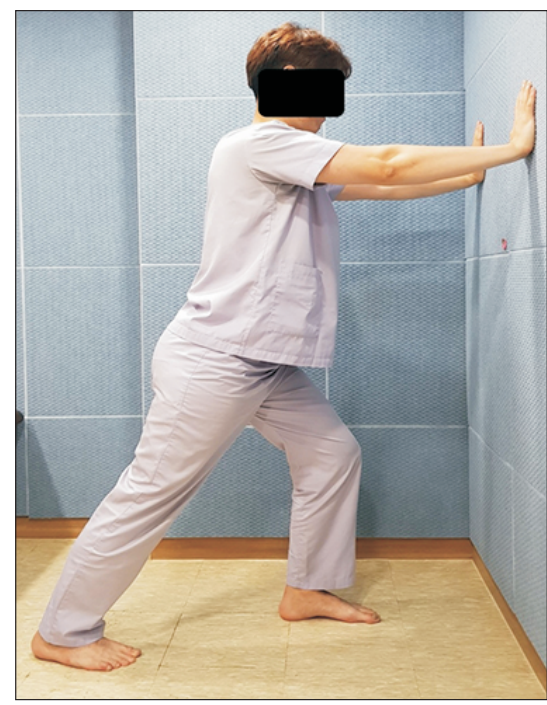

Fig. 2. Wall stretching. ence the measured outcome variables.

\section{Evaluation}

Lateral view plain radiographs were obtained, one in the neutral position and two in the maximum ankle dorsiflexion position (maximum elongation of the Achilles tendon). The length of the triceps surae was measured as the distance between the approximate insertion point of the tendon (midline of the posterior calcaneum) and the origin point of the gastrocnemius muscle (posterior surface of the lateral condyle of the femur) (Fig. 4A). The joint angle of ankle dorsiflexion was measured as the angle formed between the shaft of the fibular and the 5th metatarsal, measured on lateral view radiographs (Fig. 4B). The dorsiflexion joint angle of the ankle was calculatedusing a " $90^{\circ}$-measuring angle" formula [12]. The subjective sense during stretching was reported using a 10 -cm visual analog scale (VAS), with anchors at " 0 " (no sense of stretching) and "10" (no pain but withmaximum feeling of stretching).

The neural activity of the gastrocnemius-soleus complex during stretching, which is representative of the resistance of the muscles to the applied stretch, was measured using electromyography (EMG). EMG was performed using a Nicolet electrodiagnostic system and Syn-
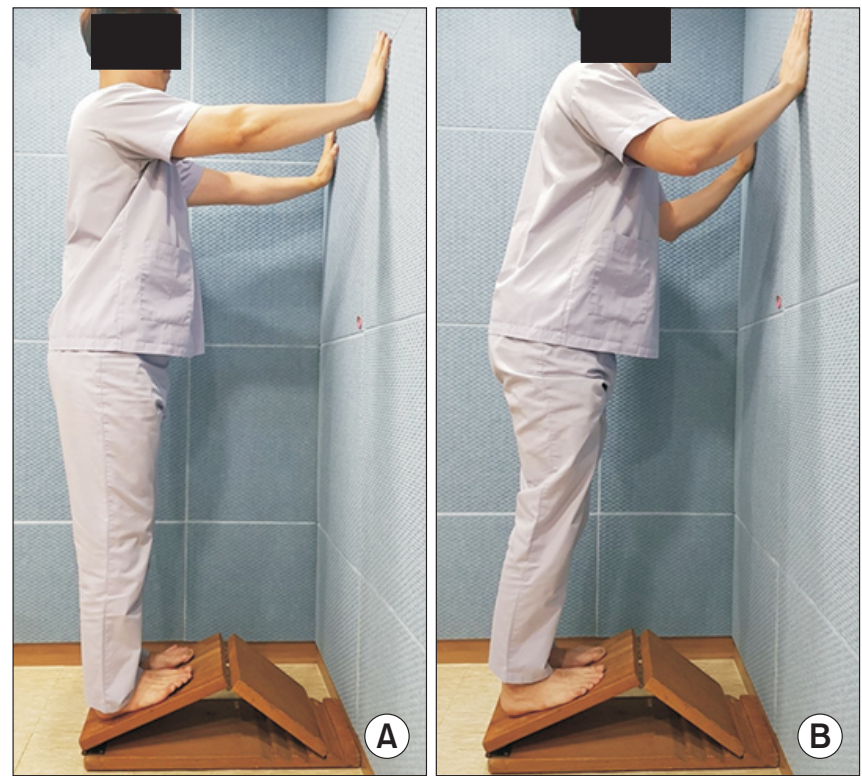

Fig. 3. Inclined board stretching. (A, B) The participant was to lean forward with their hands on the wall and then to bend their elbows until they felt a stretch in the triceps surae bilaterally. 
ergy v.22 software program (Natus Medical Inc., San Carlos, CA, USA). Surface electrodes were placed on the mid portion of the lateral belly of the gastrocnemius muscle in accordance with the SENIAM (Surface Electromyography for Noninvasive Assessment of Muscles) project guidelines [13]. Mean amplitude values were calculated for
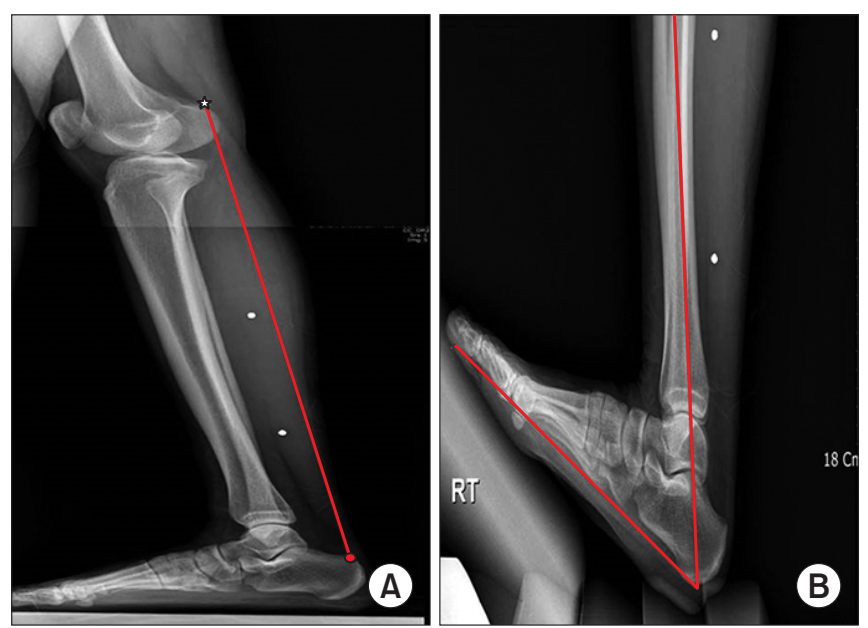

Fig. 4. Lateral view radiographs. (A) The length of the triceps surae was measured as the distance between the approximate insertion point of the tendon (circle) and the origin point of the gastrocnemius muscle (star). (B) The angle of ankle dorsiflexion was measured as the angle formed between the shaft of the fibula and the 5th metatarsal on lateral view radiographs.
10 trials and then averaged [14]. EMG was conducted in each stretching position with a 1-minute rest between trials. A lower mean EMG amplitude is indicative of greater muscle relaxation during stretching [15].

\section{Statistical analyses}

A paired t-test was performed to compare the length of the triceps surae, ankle dorsiflexion angle, mean EMG amplitude, and VAS score between the two stretching techniques. Analyses were performed using SPSS version 12.0 (SPSS Inc., Chicago, IL, USA), with the significance level set at 0.05 .

\section{RESULTS}

Of the 30 participants included in this study, 7 were men and 23 were women, with a mean age of 24.5 years, height of $162.8 \mathrm{~cm}$, and weight of $58.6 \mathrm{~kg}$. The length of the gastrocnemius-soleus complex was $40.90 \pm 3.39 \mathrm{~cm}$ after inclined board stretching and $40.17 \pm 3.41 \mathrm{~cm}$ after wall stretching, and the difference was significant $(\mathrm{p}=0.02)$ (Tables 1 and 2). Furthermore, the calculated dorsiflexion angle was significantly greater for inclined board stretching $\left(39.77^{\circ} \pm 5.02^{\circ}\right)$ than for wall stretching $\left(37.19^{\circ} \pm 5.49^{\circ}\right.$; $\mathrm{p}=0.03)$. The mean EMG amplitude was significantly lower during inclined board stretching (183.44 $\pm 59.60 \mathrm{mV}$ ) than during wall stretching $(237.16 \pm 78.83 \mathrm{mV} ; \mathrm{p}<0.01)$.

Table 1. Results after two stretching exercises

\begin{tabular}{lcc}
\hline \multicolumn{1}{c}{ Variable } & Wall stretch & Inclined board stretch \\
\hline Length of the triceps surae $(\mathrm{cm})$ & $40.17 \pm 3.41$ & $40.90 \pm 3.39$ \\
Ankle dorsiflexion ROM $\left(^{\circ}\right)$ & $37.19 \pm 5.49$ & $39.77 \pm 5.02$ \\
Mean amplitude of surface EMG $(\mathrm{mV})$ & $237.16 \pm 78.83$ & $183.44 \pm 59.60$ \\
VAS of stretching sensation & $5.97 \pm 1.88$ & $8.03 \pm 1.43$ \\
\hline
\end{tabular}

Values are presented as mean \pm standard deviation.

EMG, electromyography; ROM, range of motion; VAS, visual analog scale.

Table 2. Results of the two-tailed paired samples t-test for the two stretching exercises

\begin{tabular}{lccrr}
\multicolumn{1}{c}{ Variable } & Mean diff & SD diff & t & p-value \\
\hline Length of the triceps surae & -0.72 & 1.60 & -2.48 & 0.02 \\
Ankle dorsiflexion ROM & -2.57 & 6.05 & -2.33 & 0.03 \\
Mean amplitude of surface EMG & 53.72 & 75.68 & 3.89 & $<0.01$ \\
VAS of stretching sensation & -2.07 & 1.66 & -6.82 & $<0.01$ \\
\hline
\end{tabular}

Mean diff, mean value of wall stretching - mean value of inclined board stretching; SD, standard deviation; ROM, range of motion; EMG, electromyography; VAS, visual analog scale. 
With regard tothe subjective sensation of stretching of the triceps surae, the VAS score was significantly higher during inclined board stretching $(8.03 \pm 1.43)$ than during wall stretching $(5.97 \pm 1.88 ; \mathrm{p}<0.01)$.

In Table 2, the mean increases in ROM, length of the triceps surae, and VAS score were $2.57^{\circ}, 0.72 \mathrm{~cm}$, and 2.07 , respectively, in inclined board stretching. The mean decrease in mean EMG amplitude was $53.72 \mathrm{mV}$ during inclined board stretching. Comparison of the data of the two stretching exercises with a two-tailed paired samples t-test suggests a statistically significant difference in the mean difference between the variables $(\mathrm{p}<0.05)$.

\section{DISCUSSION}

Both techniques (wall and inclined board stretchings) increased the length of the triceps surae and ankle dorsiflexion ROM, consistent with the findings of a previous report $[2,16]$. The technique used in this study for measuring the length of the triceps surae on lateral view radiographs was previously reported as a reliable surrogate measure of the change in the length of the triceps surae muscle with stretching [17].

The increase in the length of the triceps surae induced by stretching was significantly greater with stretching on the inclined board than with wall stretching, which is indicative of the greater usefulness of stretching using an inclined board; it also translates to a difference in the range of ankle dorsiflexion between the two techniques. A key difference between the two stretching exercises is the greater ankle dorsiflexion angle during inclined board stretching than during wall stretching. In Table 2, all the variables are statistically significant, and the t value of the ROM is the largest, which is an important factor indicating the difference between the two stretching exercises. In addition, similar to previous studies, increasing the ankle dorsiflexion angle during stretching can be considered to improve the tightness of the triceps surae [2]. The contribution of the midfoot joint extension to the angle of the ankle dorsiflexion must be considered. In the study of DiGiovanni et al. [10], shoe inserts were used to minimize excessive midfoot pronation during stretching, but not in our study. Therefore, the effect of midfoot joint extension could be included while measuring the ankle joint dorsiflexion angle, which can be a limitation of this study.
Inclined board stretching was associated with a significantly lower EMG activity of the gastrocnemius-soleus complex than was wall stretching. A previous study reported that the effect of stretching on the mean EMG of both the gastrocnemius and soleus muscles with the knee in extension preferentially biasedthe stretch on the gastrocnemius [14]. In that study, stretching elicited no significant difference in the EMG activity of the soleus, with a large difference produced on the gastrocnemius according to the knee joint position. Therefore, we performed EMG on the gastrocnemius not the soleus to measure the effect of stretching in the knee extended position.

In the present study, the mean EMG was lower with inclined board stretching than with wall stretching. The greater EMG activity during wall stretchingindicates that muscle relaxation may not be sufficient to produce an effective stretching force on the triceps surae [15]. Notably, the higher EMG activity during stretching is indicative of greater muscle contraction, which would increase the strain on the muscle and tendon during stretching and could reduce the benefit of the stretch [14]. As such, the lower mean EMG activity of the lateral gastrocnemius muscle during stretching on the inclined board is indicative of the greater effectiveness of this mode of stretching than that of wall stretching in increasing the length of the triceps surae and ankle ROM.

The participants reported a greater sensation of stretching during stretching on an inclined board than during wall stretching, although this difference in subjective sensation of stretching might not necessarily indicate a more effective stretching, as it may have contributed to the pain and discomfort caused by the hard surface during inclined board stretching. Padding, particularly in the area of heel contact, might be beneficial in the future construction of inclined boards for triceps surae stretching.

In this study, two static exercises, namely, inclined board and wall stretchings, were suggested as stretching methods for the triceps surae. Although previous studies reported the benefits of wall stretching [10], the present findings favor inclined board stretching.

We provide evidencebased on an observational crosssectional study that inclined board stretching increases the length of the triceps surae, ankle ROM, and subjective sensation of stretching and lower the mean EMG 
amplitude as compared with wall stretching. Therefore, instructing individuals to perform triceps surae stretching on an inclined board might be more effective than the wall stretching technique.

\section{CONFLICT OF INTEREST}

No potential conflict of interest relevant to this article was reported.

\section{AUTHOR CONTRIBUTION}

Conceptualization: Lee JG. Methodology: Kim TH, Lee JG. Formal analysis: Kim TH, Lee JG. Project administration: Lee JG. Visualization: Kim TH, Park KD. Writing original draft: Kim TH, Lee JG. Writing - review and editing: Kim TH, Lim OK, Park KD, Lee JG. Approval of the final manuscript: all authors.

\section{REFERENCES}

1. Malhotra K, Chan O, Cullen S, Welck M, Goldberg AJ, Cullen N, et al. Prevalence of isolated gastrocnemius tightness in patients with foot and ankle pathology: a population-based study. Bone Joint J 2018;100B:94552.

2. Radford JA, Burns J, Buchbinder R, Landorf KB, Cook C. Does stretching increase ankle dorsiflexion range of motion? A systematic review. Br J Sports Med 2006;40:870-5.

3. Baumbach SF, Braunstein M, Regauer M, Bocker W, Polzer H. Diagnosis of Musculus Gastrocnemius Tightness - Key Factors for the Clinical Examination. J Vis Exp 2016;(113):53446.

4. Jeon IC, Kwon OY, Yi CH, Cynn HS, Hwang UJ. Ankledorsiflexion range of motion after ankle self-stretching using a strap. J Athl Train 2015;50:1226-32.

5. Macklin K, Healy A, Chockalingam N. The effect of calf muscle stretching exercises on ankle joint dorsiflexion and dynamic foot pressures, force and related temporal parameters. Foot (Edinb) 2012;22:10-7.

6. Thacker SB, Gilchrist J, Stroup DF, Kimsey CD Jr. The impact of stretching on sports injury risk: a systematic review of the literature. Med Sci Sports Exerc 2004;36:371-8.

7. Peters JA, Zwerver J, Diercks RL, Elferink-Gemser MT, van den Akker-Scheek I. Preventive interventions for tendinopathy: A systematic review. J Sci Med Sport 2016;19:205-11.

8. Youdas JW, Krause DA, Egan KS, Therneau TM, Laskowski ER. The effect of static stretching of the calf muscle-tendon unit on active ankle dorsiflexion range of motion. J Orthop Sports Phys Ther 2003;33:408-17.

9. Kuzma SA, McNeil SP. A comparison of prostretch versus incline board stretching on active ankle dorsiflexion range of motion. UWL J Undergrad Res 2005;8:1-6.

10. DiGiovanni BF, Nawoczenski DA, Lintal ME, Moore EA, Murray JC, Wilding GE, et al. Tissue-specific plantar fascia-stretching exercise enhances outcomes in patients with chronic heel pain: a prospective, randomized study. J Bone Joint Surg Am 2003;85:1270-7.

11. Mizuno T, Matsumoto M, Umemura Y. Viscoelasticity of the muscle-tendon unit is returned more rapidly than range of motion after stretching. Scand J Med Sci Sports 2013;23:23-30.

12. Russell JA, Shave RM, Kruse DW, Nevill AM, Koutedakis $\mathrm{Y}$, Wyon MA. Is goniometry suitable for measuring ankle range of motion in female ballet dancers? An initial comparison with radiographic measurement. Foot Ankle Spec 2011;4:151-6.

13. Hermens HJ, Freriks B, Disselhorst-Klug C, Rau G. Development of recommendations for SEMG sensors and sensor placement procedures. J Electromyogr Kinesiol 2000;10:361-74.

14. Reid D, McNair PJ, Johnson S, Potts G, Witvrouw E, Mahieu N. Electromyographic analysis of an eccentric calf muscle exercise in persons with and without Achilles tendinopathy. Phys Ther Sport 2012;13:150-5.

15. Min BC, Kim JH, Jeon KJ, Lee DH, Kim JS. EMG fatigue comparative study of stair ascending and descending. Proceedings of the Society of Korea Industrial and Systems Engineering Spring Conference; 2006 May. p. 234-7.

16. Gajdosik RL, Allred JD, Gabbert HL, Sonsteng BA. A stretching program increases the dynamic passive length and passive resistive properties of the calf muscle-tendon unit of unconditioned younger women. Eur J Appl Physiol 2007;99:449-54.

17. Foure A, Nordez A, Cornu C. Effects of eccentric training on mechanical properties of the plantar flexor muscle-tendon complex. J Appl Physiol (1985) 2013;114:523-37. 